\title{
Corredores ecológicos como ferramenta para a desfragmentação de florestas tropicais
}

\author{
Carlos Eduardo Sícole Seoane ${ }^{1}$ Vinícius Sandri Diaz², Tomaz Longhi Santos³, Luiz Claudio Maranhão Froufe ${ }^{1}$
}

1Embrapa Florestas, Estrada da Ribeira, Km 111, CP 319, CEP 83411-000, Colombo, PR, Brasil, eduardo@cnpf.embrapa.br; luisao@cnpf.embrapa.br; 2Universidade de São Paulo (USP), Escola Superior de Agricultura Luiz de Queiroz (ESALQ), Av. Pádua Dias, 11, CP 9, CEP 13418-900, Piracicaba, Brasil, Bolsista do CNPq, vinicius.diaz@usp.br; ${ }^{3}$ Acadêmico de Engenharia Florestal, Universidade Federal do Paraná (UFPR), Rua dos Funcionários, 1540, Juvevê, CEP 80035-050, Curitiba, PR, Brasil, tomazlonghi@gmail.com.

\begin{abstract}
Resumo - A expansão do uso e ocupação antrópica do espaço terrestre leva a uma severa fragmentação dos habitats naturais. Corredores ecológicos podem facilitar o deslocamento de organismos entre fragmentos de habitats e têm sido cada vez mais adotados como uma ferramenta para manter e restaurar a biodiversidade. A teoria da metapopulação é uma das bases para os atuais estudos sobre corredores ecológicos. Esses estudos ainda estão pouco desenvolvidos, especialmente em ecossistemas tropicais, carecendo de bases científicas sólidas para apoiar a sua utilidade. No entanto, os efeitos da fragmentação florestal são tão severos que é justificável o planejamento e a execução de medidas que busquem atenuá-las, apesar de não estarem disponíveis resultados de pesquisas que demonstrem a eficácia ou mesmo o acerto destas medidas.
\end{abstract}

Termos de Indexação: Corredores ecológicos, fragmentação florestal, monitoramento, permeabilidade da paisagem.

\section{Ecological corridors as an instrument for the desfragmentation of tropical forests}

\begin{abstract}
The expansion of the human land use leads to a severe fragmentation of the natural habitats. Biological corridors may facilitate the movement of organisms among habitat patches and have been frequently adopted as a tool to conserve and restore biodiversity. Metapopulation theory gives the theoretical starting point for the actual biological corridors studies, which are still in their infancy. There are not yet a solid scientific knowledge to recommend the use of biological corridors as a tool in conservation biology. Nevertheless, the effects of forest fragmentation are so severe that the planning and executing of strategies to attenuate it are justifiable, even though research results demonstrating its effectiveness and benefits are not available.
\end{abstract}

Index terms: Biological corridors, forest fragmentation, monitoring, landscape permeability.

\section{Introdução}

A expansão do uso e ocupação antrópica do solo tem como um de seus resultados uma generalizada perda e fragmentação dos habitats naturais, que podem levar ao maior evento de extinção global da história. A perda de habitats naturais tem severas consequências sobre a biodiversidade, ao afetar a taxa de crescimento populacional, diminuir o comprimento e a diversidade da cadeia trófica e alterar as interações das espécies, entre outros efeitos negativos (Forero-Medina \& Vieira, 2007). Por sua vez, a fragmentação de habitats é considerada como uma das maiores ameaças à conservação da biodiversidade ainda existente (Crooks \& Sanjayan, 2006; Brasil, 2006; Global..., 2006). Entendendo a desfragmentação de habitats como a eliminação dos efeitos negativos da perda e da fragmentação de habitats, necessita-se urgentemente de ações que visem à desfragmentação dos habitats naturais restantes. A conectividade, que é a habilidade de uma população ou espécie de se locomover entre elementos da paisagem em um mosaico de tipos de habitats (Hilty et al., 2006), é um dos processos principais para a desfragmentação florestal e, portanto, para a conservação de biodiversidade; a promoção da conectividade entre áreas a serem conservadas tem sido uma recomendação constante na literatura (Hirsh, 2003a, 2003b; Crooks \& Sanjayan, 2006; Davies \& Pullin, 2007), especialmente com a previsão de aceleração das mudanças climáticas (Philipps \& Gentry, 1994). Corredores ecológicos, elementos da paisagem que aumente a habilidade de organismos de se locomoverem entre manchas de seu habitat (Hilty et 
al., 2006), tem sido cada vez mais citados e adotados como ferramenta para alcançar a conectividade (Noss, 1987; Vieira et al., 2002; Damschen et al., 2006; Hilty et al., 2006). Os corredores ecológicos têm sido utilizados nos Estados Unidos com resultados empiricamente positivos desde o início do século 20, principalmente direcionados para o manejo de espécies de caça em áreas em desenvolvimento agrícola do centro-oeste norteamericano (Harris \& Atkins, 1991). Outras alternativas para impedir o isolamento de populações em fragmentos de habitat, como por exemplo, a translocação artificial de indivíduos, são possíveis para um número limitado de espécies, alcançando somente uma pequena fração da diversidade animal e vegetal de uma região.

O primeiro grande esforço no sentido de se construir uma teoria sobre como os organismos lidam com manchas desconectadas de habitat foi a teoria da biogeografia de ilhas, de MacArthur \& Wilson (1963, 1967), que formularam um modelo simples para explicar o número de espécies encontradas em um local, considerando que o número de espécies é o resultado de um equilíbrio entre as taxas de imigração e extinção. Tal modelo se encaixa razoavelmente bem nos dados de números de espécies, tanto em ilhas verdadeiras quanto em ilhas "virtuais", como os fragmentos florestais, e prediz que ilhas maiores terão mais espécies que ilhas menores. O modelo de MacArthur e Wilson prediz que pequenos fragmentos florestais suportarão populações menores e menos espécies que fragmentos maiores, que as comunidades insulares são mais pobres em espécies que as comunidades continentais equivalentes e que a riqueza diminui com o isolamento da ilha. Os trabalhos de Bierregaard \& Lovejoy $(1988,1989)$ confirmam a predição desse modelo, que ainda hoje é importante no estudo da ecologia de paisagens (Hilty et al., 2006). Esta teoria forneceu aos conservacionistas uma base teórica para exigir a criação de grandes reservas.

Na década de 1980, os cientistas perceberam que as atividades humanas, ao fragmentar habitats naturais, estavam transformando populações contínuas em metapopulações perturbadas. Por consequência, o conceito de metapopulação começou a substituir a teoria da biogeografia de ilhas como o paradigma predominante da biologia da conservação. Segundo Hanski \& Simberloff(1997), o modelo de metapopulação, inicialmente elaborado por Levin (1969), considera que o espaço é um mosaico, sendo possível distinguir entre manchas do ambiente adequadas para a espécie em questão e o restante do ambiente, denominado matriz, talvez adequado para a migração de indivíduos entre populações, mas inadequado para o estabelecimento populacional. Ou seja, uma metapopulação é um conjunto de populações localizadas em manchas de habitats, inseridas em uma matriz onde a migração entre as populações pode ser possível.

As dinâmicas metapopulacionais ainda incluem extinção de populações e, através da migração de indivíduos, recolonização de manchas onde houve extinção da população e colonização de partes da matriz que se tornaram manchas adequadas para a espécie (Hanski \& Gilpin, 1991). A condição básica para a persistência em longo prazo de uma metapopulação é que o número esperado de novas populações geradas por cada população durante a sua existência seja maior que um. No entanto, seja tal condição de reposição alcançada ou não, uma metapopulação de populações em uma rede mal conectada se encontra mais ameaçada que uma metapopulação mais bem conectada (Hanski, 1998). Neste sentido, com a fragmentação florestal, o isolamento de populações de uma espécie em pequenos fragmentos florestais pode levar à perda de variabilidade genética natural da espécie, principalmente devido ao gargalo genético (em curto prazo) e à deriva genética aleatória (em longo prazo) (Hall et al. 1996a; Seoane et al., 2005). Assim, muitas espécies podem estar presentes em locais que já não possuem capacidade de manter suas populações em longo prazo (Hanski, 1998).

Estas pesquisas acima descritas somam-se a outras publicações (por exemplo, Horskins et al., 2006), que levaram à formação de uma opinião comum entre os pesquisadores da biologia da conservação de que, além da urgência de se estabelecerem medidas de proteção dos remanescentes florestais, torna-se necessário restaurar a interligação entre os fragmentos florestais, para que a reprodução da fauna e da flora possa ocorrer entre indivíduos de diferentes fragmentos florestais.

Preocupações em relação a esta perda de diversidade biológica motivaram os estudiosos da biologia da conservação a discutirem as ações necessárias para aumentar o tamanho efetivo das populações (Meffe \& Carroll, 1997). Observações teóricas e empíricas demonstraram que o aumento do intercâmbio de indivíduos entre populações pode aumentar a persistência populacional local e regional, principalmente para populações pequenas e isoladas (Sjogren, 1991; Fahrig \& Merriam, 1994; Rosemberg et al., 1997). A importância 
de tal intercâmbio, tanto reduzindo as taxas de extinção quanto aumentando as taxas de colonização, tornou-se um paradigma na biologia da conservação (Simberloff, 1988; Doak \& Mills, 1994; Hilty et al., 2006).

\section{Fragmentação Florestal}

Perturbações naturais sempre resultaram em algum grau de fragmentação de habitats. No passado, áreas perturbadas geralmente eram rodeadas por uma matriz de estágios mais avançados da sucessão. Os animais simplesmente se locomoviam desviando de ilhas de perturbação (Csuti, 1991), podendo mesmo utilizar estas áreas em estágios sucessionais menos avançados como fontes de recursos. Contudo, as alterações causadas pelo homem na cobertura vegetal original reverteram esta situação matricial: as perturbações, permanentes ou temporárias, dominam a paisagem; em muitas regiões do planeta, as áreas ocupadas pelos ecossistemas naturais são pequenas manchas rodeadas por áreas ocupadas e utilizadas pelas atividades antrópicas. Este processo é denominado fragmentação, definida como a transformação de um habitat contínuo em manchas de habitat, que variam em tamanho e forma (Fahrig, 2003). Estas pequenas manchas de ecossistemas naturais, cercadas pelas atividades humanas, também são chamadas de fragmentos ou remanescentes, e as atividades humanas, que dominam a paisagem, de matriz antrópica. Quando o ecossistema natural é uma floresta, estas pequenas manchas de ecossistemas naturais são denominadas fragmentos florestais, a exemplo do que ocorre atualmente com os remanescentes de Mata Atlântica.

Vários são os efeitos negativos da fragmentação florestal sobre a sobrevivência da fauna e da flora nativa; entre estes, um que merece destaque é o isolamento reprodutivo. $\mathrm{O}$ mosaico de habitats que é gerado pelas atividades humanas, incluindo manchas de ecossistemas nativos, fragmentos antropogênicos e áreas agrícolas e urbanas, apresenta probabilidades desprezíveis de dispersão e estabelecimento de indivíduos de parte da fauna (Janzen, 1990; Santos Filho, 1995). A fragmentação pode levar a modificações nos padrões de polinização e dispersão de sementes e, assim, acarretar em modificações na diversidade e estrutura genética das espécies tanto animais quanto vegetais, pois: a) altera a composição dos agentes polinizadores e dispersores, podendo alterar seus comportamentos; b) as espécies arbóreas das florestas tropicais são em sua maioria alógamas (Bawa et al., 1985a), sendo sua polinização fundamentalmente realizada por animais (Bawa et al., 1985b), destacando-se insetos, morcegos e aves, muitas espécies arbóreas tropicais têm dispersão de sementes realizada por animais (Howe, 1984; Seoane et al., 2005). Os indivíduos isolados muitas vezes não conseguirão se reproduzir e, caso consigam, a reprodução acontecerá entre poucos indivíduos presentes no fragmento florestal, trazendo um efeito negativo para a sobrevivência da espécie denominado "depressão endogâmica", que poderá levar à perda da adaptabilidade da espécie ou mesmo à extinção (Young et al., 2000). Há na literatura uma grande quantidade de estudos sobre os efeitos do isolamento reprodutivo causado pela fragmentação florestal (Hall et al., 1996; Tabarelli \& Gascon, 2005; Seoane et al., 2000, 2005).

Outro efeito da fragmentação florestal está relacionado ao tamanho da área. As espécies que demandam uma maior área para sua sobrevivência são muitas vezes as primeiras a serem extintas. Soulé (1991) lembra que espécies em grandes populações, mesmo em pequenas áreas, têm uma grande probabilidade de persistência em longo prazo, mesmo sem dispersão entre fragmentos de habitat, assim, argumentando contra a necessidade de corredores de movimento para tais animais. Contudo, existem consequências evolucionárias do isolamento que podem surgir em uma escala de tempo de maior amplitude, e tais consequências seriam importantes o bastante para haver a preocupação de evitá-las, através da manutenção ou recuperação da conectividade entre fragmentos de habitats. Uma delas surge do fato de que qualquer população tem uma dada probabilidade de sobrevivência por um determinado tempo, e passado esse prazo, todas as populações provavelmente serão extintas, caso não ocorra a recolonização por populações vizinhas. Assim, se tal recolonização não ocorrer, a espécie estará, a priori, erodida geneticamente e, depois, extinta localmente. Portanto, uma questão básica para a conservação das florestas tropicais é: "que tamanho e forma deve ter uma reserva para ser eficiente na preservação de espécies?" As estratégias de manejo e conservação direcionadas para preservar a variação genética intrapopulacional das espécies tropicais tem que considerar a manutenção de grandes populações e/ou metapopulações, o que requer grandes áreas destinadas à conservação (Kageyama \& Gandara, 1993; Hilty et al., 2006). 
Baseando-se principalmente nos dados disponíveis para Hevea brasiliensis (seringueira), espécie arbórea da Amazônia com baixíssima densidade de indivíduos por hectare, Kageyama \& Gandara (1993) discutiram sobre o tamanho mínimo de área para conservação dos recursos genéticos dessa espécie tropical. Considerandose que um número mínimo empírico de 500 indivíduos não endogâmicos é necessário para representar uma população para a conservação (Frankel \& Soulé, 1981), uma população desta espécie ocupa uma área de 25 mil hectares. Como para cada unidade de área onde ocorre uma população de $H$. brasiliensis existe intercaladamente uma média de três unidades equivalentes sem a espécie, onde ocorrem outras populações de espécies de baixa densidade, com dinâmicas populacionais semelhantes às de $H$. brasiliensis, uma amostra da diversidade arbórea amazônica ocupa cerca de 100 mil hectares. Considerando-se que, na falta de dados específicos, um mínimo de cinco populações seria o suficiente para formar uma metapopulação (Hanski \& Gilpin, 1991) e assim viabilizar as espécies em longo prazo, a área total para manter essas espécies passa para $500 \mathrm{mil}$ hectares (Kageyama \& Gandara, 1993). Considerando que, em termos de espaço, as espécies mais exigentes são as de baixa densidade, uma área que compreenda metapopulações daquelas espécies de menor densidade deve também compreender metapopulações daquelas de maior densidade. Assim, uma área de 500 mil hectares garantiria a perpetuação da biodiversidade arbórea local, isto se considerarmos uma área com forma arredondada, garantindo um efeito de borda reduzido (Meffe \& Carrol, 1997). A maior parte da biodiversidade arbórea tropical é composta por espécies de densidade baixa (Kageyama \& Gandara, 1993). Se considerarmos estes 500 mil hectares também como sendo a área mínima para garantir a perpetuação da biodiversidade arbórea da Mata Atlântica, é enorme a ameaça à biodiversidade deste bioma representada pela atual fragmentação florestal ali ocorrente, pois poucas são as áreas de Mata Atlântica que alcançam um tamanho perto de 500 mil hectares (Fundação S.O.S Mata Atlântica, 2008).

\section{Migração, fluxo gênico e fragmentação florestal}

As populações de quase todas as espécies exibem algum grau de diferenciação genética entre locais geográficos, simplesmente por que os juvenis geralmente tendem a iniciar seu ciclo de vida perto uns dos outros e de seus pais, e pares que se cruzam poucas vezes representam amostras ao acaso a partir da área geográfica onde a espécie ocorre (Ehrlich \& Raven, 1969). Além de muitas espécies terem uma estrutura genética microgeográfica, em escalas geográficas amplas, geralmente, aparecem diferenciações adicionais, devido à estrutura espacial de habitats, isolamento por distância e outros fatores (Avise, 1994). Assim, as populações de muitas espécies variam na composição genética, às vezes em áreas espaciais na grandeza de quilômetros ou de metros (Stewart et al., 1990).

Um importante desafio para os cientistas é descrever as arquiteturas genéticas populacionais dentro de espécies e identificar e ordenar as forças evolucionárias responsáveis por cada caso. Em termos gerais, tais forças envolvem migração ou fluxo gênico, deriva genética aleatória, várias formas de seleção natural, divergência mutacional e oportunidade de recombinação genética mediadas pelo sistema de cruzamento. O fluxo gênico é definido como a transferência de material genético entre populações, resultante do movimento de indivíduos ou de seus gametas. Geralmente, o fluxo gênico é expresso como a taxa de migração $(m)$, definida como a proporção de alelos em uma população em cada geração que é de origem migrante (Avise, 1994). Contrastando com forças disruptivas, o fluxo gênico oriundo da migração é o movimento de organismos entre populações, funcionando como uma cola genética que segura as populações geneticamente juntas, na forma de uma metapopulação. A migração tende a homogenizar populações e a aumentar a variação dentro de populações. É notável como pouca migração é necessária para evitar uma significativa divergência genética entre populações (Hartl \& Clark, 1997; Hamrick \& Nason, 2000).

O monitoramento da migração durante uma ou poucas gerações geralmente é baseado em marcadores físicos, marcadores fluorescentes, radiotelemetria e caracteres morfológicos, podendo também empregar marcadores moleculares para efetuar uma análise de parentesco (Young et al., 2000; Seoane et al., 2005), sendo a maior limitação para esta última metodologia a impraticabilidade de monitorar precisamente movimentos em longa distância. Tal fluxo gênico de longa distância, mesmo que raro, pode ter uma influência homogeneizadora significativa na estrutura genética das espécies (Avise, 1994). Por outro lado, os migrantes talvez não sejam tolerados pelos coespecíficos locais e também podem não ser integrados nas populações receptoras se 
no local está ocorrendo falta de recursos ou espaço, o que pode ser bem comum em fragmentos florestais. Portanto, a detecção de migração de indivíduos não deve ser considerada diretamente ligada ao fluxo gênico (Slatkin, 1994). Ainda, com o bloqueio de rotas de migração, muitas das espécies não possuem mais as condições de equilíbrio em sua genética de populações, pois sua estrutura genética pode ter sido alterada, populações podem ter sido extintas, e as remanescentes podem estar fragmentadas e pequenas. Tais fatores podem levar ao isolamento de populações que naturalmente trocavam genes com outras populações.

\section{Corredores Ecológicos}

Desde a década de 1970, os corredores ecológicos são citados como parte de estratégias para a conservação de ecossistemas fragmentados (Diamond, 1975; Wilson \& Willis, 1975; Meffe \& Carroll, 1997). Desde então, vários estudos citam os potenciais benefícios dos corredores; entre eles, destaca-se a possibilidade de deslocamento das espécies nativas (Noss, 1983; Noss \& Harris, 1986; Hilty et al., 2006; Crooks \& Sanjayan, 2006; Weldon, 2006). Os corredores ecológicos já fazem parte da legislação ambiental brasileira, desde a Lei Federal No $9.985 / 2000$, que estabeleceu o Sistema Nacional de Unidades de Conservação (SNUC), em cujo artigo $2{ }^{\circ}$ se define corredores ecológicos: porções de ecossistemas naturais ou seminaturais, ligando unidades de conservação, que possibilitam entre elas o fluxo de genes e o movimento da biota, facilitando a dispersão de espécies e a recolonização de áreas degradadas, bem como a manutenção de populações que demandam, para sua sobrevivência, áreas com extensão maior do que aquela das unidades individuais".

Há muitas definições de corredores ecológicos na literatura científica, além da citada por Hilty et al. (2006). Estas definições podem ser agrupadas em duas categorias de acordo ao que se referem, se à estrutura ou à funcionalidade do corredor na paisagem. As definições estruturais, também chamadas de definições de padrão, salientam a linearidade da forma do corredor ecológico e sua distinção fisionômica em relação à matriz; e as definições funcionais, também chamadas de definições de processo, referem-se aos elementos na paisagem e sua suposta facilitação ao movimento de organismos. Da mesma forma que para os corredores, há dois tipos de conectividade considerados na literatura: a conectividade estrutural, que considera somente os aspectos físicos da paisagem, e a conectividade funcional, que considera a interação da paisagem e dos organismos nela presentes, sendo dependente da capacidade de dispersão do organismo e sua capacidade de usar a matriz e o habitat (Forero-Medina \& Vieira, 2007).

Há efeitos negativos potenciais associados aos corredores ecológicos, que podem ser agrupados àqueles relacionados aos efeitos de borda, à deriva da comunidade, facilitação de movimento para espécies invasoras, impactos demográficos, impactos genéticos e mesmo aos impactos econômicos na implantação de corredores ecológicos (Hilty et al., 2006). No entanto, muitos destes efeitos negativos são esperados principamente em elementos da paisagem que não foram criados para servirem como corredores ecológicos, como cercas vivas, quebra-ventos e outras construções humanas. Estes elementos da paisagem apresentam benefícios paisagísticos e antrópicos e, em algumas circunstâncias, podem estar funcionando como corredores ecológicos; no entanto, não são invariavelmente corredores ecológicos. É ainda fundamental reconhecer que, para uma espécie, o que é propício como corredor pode não ser para uma outra, podendo mesmo se constituir em uma barreira. Além disto, ao facilitar a movimentação de uma determinada espécie, o corredor pode estar trazendo efeitos negativos sobre outra, como em uma relação predador-presa (Weldon, 2006).

Segundo Primack \& Rodrigues (2001), a conservação de áreas grandes e alongadas é uma idéia biologicamente interessante, mas representa ou um custo político que poucos governos estão dispostos a pagar, ou um volume de recursos difícil de obter. Dentro desta percepção, além das definições de corredor apresentadas acima, que se referem a corredores ecológicos sensu strictu, há na literatura especializada e, principalmente, nas estatégias governamentais, um outro tipo de corredor ecológico, baseado na aumento de permeabilidade da matriz para a movimentação das espécies (Rouget et al., 2006), que aqui serão denominados de corredores ecológicos institucionais. Trata-se de levar para o mosaico de elementos encontrados na paisagem determinadas mudanças no uso da terra, planejadas para manter ou recuperar a conectividade entre fragmentos de habitats, formando um mosaico planejado de áreas exploradas, seminaturais e protegidas com função tanto econômica quanto ecológica (Noss, 1983; Noss \& Harris, 1986). Pe'er et al. (2005) afirmam que, apesar de os corredores 
ecológicos geralmente serem percebidos como elementos da paisagem claramente visíveis, encontrados em uma matriz hostil, a biota muitas vezes irá canalizar seus movimentos em rotas específicas que, à primeira vista, parecem iguais à paisagem circundante. Os corredores intitucionais seriam, assim, uma alternativa talvez mais viável, em termos políticos e financeiros.

Nos corredores institucionais, o aumento da permeabilidade de matriz seria alcançado ao implantar, na matriz antrópica, práticas agrícolas e usos de solo que sejam mais adequados para a passagem da biota por ali como, por exemplo, a diminuição do uso de agrotóxicos nas lavouras. Tal é o fundamento de alguns programas governamentais em execução no Brasil, como o Corredores Ecológicos, do Governo Federal Brasileiro (Arruda, 2006a, 2006b; Ibama, 2007), que objetiva contribuir para a conservação efetiva da biodiversidade do país ao propor a criação de corredores intitucionais na Floresta Amazônica e na Mata Atlântica, pretendendose manter ou restaurar a conectividade da paisagem através da gestão do território em escala regional (Ibama, 2007). Para a consecução do Projeto, foram indicados sete grandes corredores, que serviriam a cerca de $25 \%$ das florestas tropicais úmidas do Brasil, sendo cinco deles localizados na Amazônia (Corredor Central da Amazônia, Corredor Norte da Amazônia, Corredor Oeste da Amazônia, Corredor Sul da Amazônia, Corredor dos Ecótonos Sul-amazônicos) e dois, na Mata Atlântica (Corredor Central da Mata Atlântica e Corredor Sul da Mata Atlântica ou Corredor da Serra do Mar) (Brasil, 2006).

\section{Corredores ecológicos como ferramenta para a desfragmentação de habitats}

Harris e Atkins (1991) sustentam que há duas maneiras de reduzir o efeito da fragmentação de habitats: aumentar o tamanho de áreas de conservação vizinhas até que elas formem uma só, recuperar ou criar corredores ecológicos entre tais áreas. A estratégia de aumentar o tamanho das áreas de conservação tem parcialmente funcionado, por exemplo, na Flórida, EUA, onde parte da arrecadação do imposto público tem sido revertido para a compra de áreas de interesse estratégico para a conservação biológica (Mann \& Plummer, 1993), mas tal êxito é uma exceção. No Brasil, é impraticável, tanto econômica quanto socialmente, ter êxito em implantar uma estratégia que vise a um aumento das Unidades de Conservação de Proteção Integral e que se consiga áreas de proteção integral grandes o suficiente para conservar a biodiversidade das florestas, especialmente no caso da Mata Atlântica. Sendo assim, o maior argumento em favor da implantação e restauração de corredores ecológicos nas florestas tropicais é o fato de eles serem, se não a única, a estratégia mais viável para a desfragmentação florestal.

Desde a década de 1990, pesquisas empíricas tem gerado evidências para a viabilidade do uso de corredores ecológicos como ferramenta para a desfragmentação florestal, principalmente, ao mostrarem que corredores ecológicos aumentam a locomoção em paisagens fragmentadas para muitas espécies de menor tamanho corporal e de tempo de vida mais curto (Haddad et al., 2003; Haddad \& Tewksbury, 2006). Davies \& Pullin (2007) revisaram os resultados de 26 estudos sobre a utilização de cercas-vivas como corredores biológicos e concluiram que, apesar das evidências empíricas ainda serem insuficientes para avaliar a efetividade, há indicações de que espécies utilizam as cercas-vivas para sua movimentação. Além das cercas-vivas, alguns trabalhos encontraram evidências de que outras formas de corredor ecológico são funcionais (Mansergh \& Scotts, 1989; Dixon et al., 2006; Damschen et al., 2006), enquanto outros não encontraram evidências (Horskins et al., 2006). Estudos também apontam que a reinterligação de fragmentos de habitats também podem exercer efeitos negativos sobre a conservação da biodiversidade (Simberloff \& Cox, 1987; Csuti, 1991; Soulé, 1991; Weldon, 2006).

Vários aspectos devem ser considerados sobre estas evidências da funcionalidade dos corredores ecológicos. Primeiramente, é a falta de evidência para espécies maiores e de ciclo de vida mais longo, para as quais as dinâmicas ambientais operam em uma escala de tempo de década para séculos (Csuti, 1991). Assim, para estas espécies, a contribuição de corredores ecológicos implantados para a desfragmentação florestal só poderá ser inteiramente entendida em longo prazo. Segundo, os efeitos dos corredores ecológicos sobre a viabilidade populacional são poucos estudados (Haddad \& Tewksbury, 2006). Terceiro, ainda há pouco conhecimento sobre os efeitos dos corredores ecológicos na estrutura e diversidade das comunidades.

Um quarto ponto importante é que, na maioria dos estudos já realizados sobre o tema, os corredores ecológicos são remanescentes dos ecossistemas naturais originais (Powell \& Bjork,1995; Seoane et al., 2005) e 
não ecossistemas antropicamente restaurados. Ou seja, poucos estudos sobre corredores ecológicos se focaram sobre a eficiência de um corredor ecológico implantado pelo homem e, quando o fazem, em muitos deles, as condições experimentais são muito diferentes dos habitats naturais da espécie em questão (por exemplo, Coffman et al., 2001). Um quinto ponto a ser considerado é que a grande maioria dos estudos que mostraram evidências da eficácia de corredores ecológicos não foram realizados na floresta tropical.

Hilty et al (2006) enfatizam a necessidade de se considerar a importância funcional do corredor em detrimento de sua estrutura, já que o objetivo primeiro do corredor ecológico é a facilitação da movimentação das espécies através dele; a implantação de um corredor ecológico pode levar imediatamente a uma maior conectividade estrutural, mas nem sempre a conectividade funcional é alcançada (Chetkiewicz et al., 2006; Forero-Medina \& Vieira, 2007). As estratégias de conservação de áreas muito fragmentadas, como, por exemplo, a Mata Atlântica, dependem de informações sobre como os processos ecológicos e evolutivos podem ser mantidos nos fragmentos pequenos, descontínuos e alterados que ainda persistem. Um desses processos é o movimento dos organismos entre os fragmentos, influenciado pelo uso da matriz, mortalidade durante a dispersão e capacidade de percepção de fragmentos à distância, entre outros (Forero-Medina \& Vieira, 2007). Assim, é impossível extrapolar que há conectividade funcional pelos resultados únicos da conectividade estrutural, como normalmente ocorre com a instalação de corredores ecológicos.

Um ponto em discussão na literatura é a largura mínima que torna um corredor ecológico eficiente, em função do efeito de borda adentrar nos corredores ecológicos (Janzen, 1986; Csuti, 1991). Efeitos de borda geralmente são encontrados a $200 \mathrm{~m}$ da borda física (Temple \& Cary, 1988) e às vezes a $600 \mathrm{~m}$ da borda (Wilcove et al., 1986), inclusive em florestas tropicais (Schroth et al., 2004). Assim, utilizando a estimativa de $600 \mathrm{~m}$, apenas um corredor com largura maior que 1,2 km conterá habitat livre dos efeitos da borda. No entanto, Primack \& Rodrigues (2006) afirmam que os principais efeitos de borda teriam apenas $35 \mathrm{~m}$ de largura.

Quanto aos corredores institucionais, ainda poucos estudos abordaram as diferenças das distintas matrizes antrópicas, em relação à sua permeabilidade ao fluxo gênico das espécies nativas (Laurance, 2004). Ainda não existem hipóteses formais sobre a permeabilidade de matrizes dominadas por diferentes usos do solo; um plantio de eucalipto, situado entre dois fragmentos florestais, pode ser considerado um corredor ecológico? E uma plantação de soja? E sistemas silvipastoris? Intuitivamente, pode-se apontar este uso do solo ou aquele outro como mais permeáveis para as espécies, mas carecemos de dados obtidos através de métodos científicos, e mesmo de hipóteses. Exceções a este quadro são a ainda não testada 'hipótese da matriz agroflorestal' de Schroth et al. (2004), segundo a qual haverá uma maior dispersão de sementes através de sistemas silvipastoris do que através de pastos limpos ou monoculturas agrícolas, e a pesquisa de Castellon \& Sieving (2006), afirmando que uma matriz arbustiva pode ser tão facilitadora para a movimentação da fauna quanto um corredor ecológico sensu stritu.

\section{Considerações finais}

Os objetivos de implantação e restauração de corredores ecológicos devem ser claramente voltados para a funcionalidade destes. Contudo, como os estudos sobre corredores ecológicos ainda são incipientes, atualmente carecemos de bases científicas sólidas para apoiar seu uso, ficando as iniciativas de manutenção, restauração e implantação de corredores ecológicos sujeitas à intuição dos técnicos envolvidos, ao jogo político e às críticas. Por outro lado, os efeitos da fragmentação da floresta tropical têm se mostrado tão severos que é justificável o planejamento e a execução de medidas que busquem atenuá-las, apesar de não estarem disponíveis resultados de pesquisas que demonstrem a eficácia ou mesmo o acerto destas medidas.

É necessário que os formuladores e implantadores das políticas públicas em torno do tema "corredores ecológicos" incluam em seus planos uma constante revisão e reformulação destas políticas baseadas nos novos resultados das pesquisas realizadas sobre o tema. Neste sentido, mais preocupante do que o fato de não haver demonstrações da eficácia das medidas sendo implantadas, é o fato de que não seja do conhecimento dos responsáveis por estas políticas a carência de bases científicas sólidas para apoiar o uso de corredores ecológicos. 


\section{Referências}

ARRUDA, M. B. Corredores ecológicos no Brasil: o enfoque ecossistêmico na implementação da Convenção da Biodiversidade. In: ARRUDA, M. B. (Org.). Gestão Integrada de Ecossistemas Aplicada a Corredores Ecológicos. IBAMA, Brasília, p.19-54, 2006a.

ARRUDA, M. B. (Org.). Gestão Integrada de Ecossistemas Aplicada a Corredores Ecológicos. IBAMA, Brasília. $2006 \mathrm{~b}$. $472 \mathrm{p}$.

AVISE, J. C. Molecular Markers, Natural History and Evolution. Chapman and Hall, New York, NY. 1994. 511 p.

BAWA, K. S.; PERRY D. R; BEACH, J.H. Reproductivebiology of tropical lowland rain-forest trees: Sexual systems and incompatibility mechanisms. American Journal of Botany. v. 72, n. 3, p. 331-345, 1985

BIERREGAARD, R. O. J.; LOVEJOY, T. E. Birds in Amazonian forest fragments: Effects of insularization. In: Ouellet, H. (ed.) Acta XIX Congresss International of Ornithology, University of Ottawa Press, Ottawa, v. 2, p. 1564-1579, 1988.

BIERREGAARD, R. O. J.; LOVEJOY, T. E. Effects of forest fragmentation on Amazonian understory bird communities. Acta Amazónica, v. 19, p. 215-241, 1989

BRASIL. Ministério do Meio Ambiente. O corredor central da mata atlântica: uma nova escala de conservação da biodiversidade. Brasília: Ministério do Meio Ambiente, 2006. $52 \mathrm{p}$.

CASTELLON, T. D.; SIEVING, K. E. An experimental test of matrix permeability and corridor use by an endemic understory bird. Conservation Biology. v. 20, n. 1, p. 135-145, 2006.

COFFMAN, C.J., NICHOLS, J.D.; POLLOCK, K.G. Population dynamics of Microtus pennsylvanicus in corridor-linked patches. Oikos, v. 93, n. 1, p. 3-21, 2001. DOI: 10.1034/j.16000706.2001.930101.x

CHETKIEWICZ, C. L. B.; CLAIR C.C.S.; BOYCE, M.S. Corridors for conservation: Integrating pattern and process. Annual Review of Ecology Evolution and Systematics .v. 37, p. 317-342, 2006.

CROOKS, K. R.; SANJAYAN, M. (eds.). Connectivity Conservation. Cambridge University Press, 710 p. 2006.

CSUTI, B. Introduction. In: Hudson, W. E. Landscape Linkage and Biodiversity. Island Press, DC, p. 81-90, 1991.

DAMSCHEN, E. I.; HADDAD N.M.; ORROCK J.L.; TEWKSBURY, J.J.; LEVEY, D.J. Corridors increase plant species richness at large scales. Science. v. 313, n. 5791, p. 1284-1286, 2006.

DAVIES, Z. G.; PULLIN, A. S. Are hedgerows effective corridors between fragments of woodland habitat? An evidence-based approach. Landscape Ecology .v. 22, n. 3, p. 333-351, 2007.

DIAMOND, J. M. The island dilemma: lessons of modern biogeography studies for the design of natural reserves. Biological Conservation, v. 7, p. 129-146, 1975.
DIXON, J. D.; OLI, M. K.; WOOTEN, M. C.; EASON, T. H.; MCCOWN, J.W.; PAETKAU, D. Effectiveness of a regional corridor in connecting two Florida black bear populations. Conservation Biology .v. 20, n. 1, p. 155-162, 2006.

DOAK, D. F.; MILLS, L. S. A useful role for theory in conservation. Ecology .v. 75, n. 3, p. 615-626, 1994.

EHRLICH, P. R.; RAVEN, P. H. Differentiation of populations. Science .v. 167, n. 3925, p. 1636-1637, 1970.

FAHRIG, L. Effects of habitat fragmentation on biodiversity. Annual Review of Ecology Evolution and Systematics .v. 34, p. 487-515, 2003.

FAHRIG, L.; MERRIAM, G. Conservation of fragmented populations. Conservation Biology .v. 8, n. 1, p. 50-59, 1994.

FORERO-MEDINA, G. ; VIEIRA, M. V. Conectividade funcional e a importância da interação organismo-paisagem. Oecologia Brasiliensis, v. 11, n. 4, p. 493-502, 2007.

FRANKEL, O.; SOULÉ, M. Conservation and evolution. Cambridge University Press, Cambridge, 1981. 327 p.

FUNDAÇÃO S.O.S. MATA ATLÂNTICA (São Paulo, SP). Atlas dos remanescentes florestais da Mata Atlântica, período de 2000 a 2005. São Paulo: Fundação S.O. S. Mata Atlântica / Instituto Nacional de Pesquisas Espaciais, 2008. Disponível em $<$ http://www.sosma.org.br/index.php?section=atlas\&action=atl as>. Acesso em: 20 jun. 2008

GLOBAL Biodiversity Outlook 2 (English). Secretariat of the Convention on Biological Diversity. Montreal, Quebec, 2006. 81 p.

HADDAD, N. M.; BOWNE, D.R.; CUNNINGHAM, A.; DANIELSON, B.J.; LEVEY, D.J.; SARGENT, S. Corridor use by diverse taxa. Ecology .v. 84, n. 3, p. 609-615, 2003.

HADDAD, N. M.; TEWKSBURY, J. J. Impacts of corridors on populations and communities. In: CROOKS, K.; SANJAYAN, M. (eds.). Connectivity Conservation. Cambridge University Press, Cambridge, England. p. 2006. 390-415.

HALL, P.; WALKER, S.; BAWA, K. Effect of forest fragmentation on genetic diversity and mating system in a tropical tree, Pithecellobium elegans. Conservation Biology .v. 10, n. 3, p. 757-768, 1996a.

HALL, R. T.; MILLER, M.; KHATTAK, A. Evaluating the effectiveness of integrated traffic corridors: Concept and practice. Its Journal .v. 3, n. 1, p. 49-67, 1996 b.

HAMRICK, J. L.; NASON, J.D. Gene flow in forest trees. In: YOUNG, A, BOSHIER, D, BOYLE, T. (Eds.). Forest Conservation Genetics: Principles and Practice, CABI Publishing, Wallingford, p. 81-90, 2000.

HANSKI, I.; GILPIN, M. Metapopulation dynamics - briefhistory and conceptual domain. Biological Journal of the Linnean Society .v. 42, n. 1-2, p. 3-16, 1991.

HANSKI, I.; SIMBERLOFF, D. The metapopulation approach, its history, conceptual domain, and application to conservation. In: HANSKI, I. A.; GILPIN, M. E. (eds.), Metapopulation Biology. Academic Press, San Diego, Californina, p. 5-26, 1997. 
HANSKI, I. Metapopulation dynamics. Nature .v. 396, n. 6706, p. 41-49, Nov 1998.

HARRIS, L. D.; ATKINS, K. Faunal movement corridors in florida. Landscape Linkages and Biodiversity, 1991.

HARTL, D. L.; CLARK, A. G. Principles of Population Genetics. Sinauer Associates., New York, 1997. 565 p.

HILTY, J. A.; LIDICKER, W. Z.; MERENLENDER, A. M. Corridor Ecology: the science and practice of linking landscapes for biodiversity conservation. Island Press, 2006. $325 \mathrm{p}$.

HIRSH, A. Habitat fragmentation and priority areas for primate conservation in the Rio Doce Basin, Minas Gerais. Neotropical Primates, v. 11 n. 3, p. 195-196, 2003.

HIRSH, A. Avaliação da Fragmentação do Habitat e Seleção de Áreas Prioritárias para a Conservação dos Primatas da Bacia do Rio Doce, Minas Gerais, Através da Aplicação de um Sistema de Informações Geográficas. 2003. 277 f. Tese (Doutorado em Ecologia, Conservação e Manejo de Vida Silvestre) - Universidade Federal de Minas Gerais, Belo Horizonte.

HORSKINS, K.; MATHER P. B.; WILSON, J. C. Corridors and connectivity: when use and function do not equate. Landscape Ecology, v. 21, n. 5, p. 641-655, jul. 2006.

HOWE, H. F. Implications of seed dispersal by animals for tropical reserve management. Biological Conservation .v. 30, n. 3, p. 261-281, 1984.

IBAMA. Corredores Ecológicos: experiências em planejamento e implementação. Ministério do Meio Ambiente, Secretaria de Biodiversidade e Florestas. Brasília, 2007. 57 p.

JANZEN, D. H. An abandoned field is not a tree fall gap. Vida Silvestre Tropical, v. 2, p. 64-67, 1990.

JANZEN, D. H. Chihuahuan desert nopaleras - defaunated big mammal vegetation. Annual Review of Ecology and Systematics, v. 17, p. 595-636, 1986.

KAGEYAMA, P. Y.; GANDARA, F. B. Dinâmica de populações de espécies arbóreas: Implicações para o manejo e a conservação. In: SIMPÓSIO DE ECOSSISTEMAS DA COSTA BRASILEIRA, 3., 1993 Anais. São Paulo: Academia de Ciências do Estado de São Paulo, 1993. p. 1-9.

LAURANCE, W. F. Landscape connectivity and biological corridors. In: SCHROTH, G.; FONSECA, G.A.B.; HARVEY, C. A.; GASCON, C.; VASCONCELOS, H. L.; IZAC, A. N. (eds). Agroforestry and Biodiversity Conservation in Tropical Landscapes. Island Press, p. 50:64, 2004.

LEVIN, B. R. A model for selection in systems of species competitionin. In: HEINMETS, F. (ed.). Concepts and Models in Biomathematics. New York, M Dekker, 1969. p. 237-273.

MacARTHUR, R. H.; WILSON, E. O. Equilibrium-theory of insular zoogeography. Evolution .v. 17, n. 4, p. 373-378, 1963.

MacARTHUR, R. H.; WILSON, E. O. The Theory of Islands Biogeography. Princeton Press, 1967. 224 p.

MANN, C. C.; PLUMMER, M. L. The high cost of biodiversity. Science .v. 260, n. 5116, p. 1868-1871, jun. 1993.
MANSERGH, I. M.; SCOTTS, D. J. Habitat continuity and social-organization of the mountain pygmy-possum restored by tunnel. Journal of Wildlife Management, v. 53, n. 3, p. 701-707, jul. 1989.

MEFFE, G. K.; CARROLL, C. R. Principles of Conservation Biology. Sinauer Associates, 1997. 600 p.

NOSS, R. F. A regional landscape approach to maintain diversity. Bioscience, v. 33, n. 11, p. 700-706, 1983.

NOSS, R. F. Corridors in real landscapes: a reply to Simberloff \& Cox. Conservation Biology, v. 1, p. 159-164, 1987.

NOSS, R. F.; HARRIS, L. D. Nodes, networks, and mums preserving diversity at all scales. Environmental Management, v. 10, n. 3, p. 299-309, 1986.

PE'ER, G.; SALTZ, D.; FRANK, K.; Virtual corridors for conservation management. Conservation Biology, v. 19, n. 6, p. 1997-2003, 2005.

PHILLIPS, O. L.; GENTRY, A. H. Increasing turnover through time in tropical forests. Science, .v. 263, n. 5149, p. 954-958, 1994.

POWELL, G. V. N.; BJORK, R. Implications of intratropical migration on reserve design: a case-study using pharomachrusmocinno. Conservation Biology, v. 9, n. 2, p. 354-362, 1995.

PRIMACK, R. B.; RODRIGUES, E. Biologia da conservação. Londrina: Midiograf, 2001. 312 p.

ROSEMBERG, D. K.; NOON, B. R.; MESLOW, E. C. Biological corridors: Form, function, and efficacy. Bioscience, v. 47, n. 10, p. 677-687, 1997.

ROUGET, M.; COWLING, R. M.; LOMBARD, A. T.; KNIGHT, A. T.; GRAHAM, I. H. K. Designing large-scale conservation corridors for pattern and process. Conservation Biology, v. 20, n. 2, p. 549-561, 2006.

SANTOS FILHO, P. S. Fragmentação de habitats: implicações para a conservação in situ. Oecologia Brasiliensis, v. 1., n. 1, p. 365-393, 1995.

SCHROTH, G.; FONSECA, G. A. B.; HARVEY, C. A.; GASCON, C.; VASCONCELOS, H. L.; IZAC, A.N. (eds). Agroforestry and Biodiversity Conservation in Tropical Landscapes. Island Press. 2004. 523 p

SEOANE, C. E. S.; SEBBENN, A. M.; KAGEYAMA, P. Y. Efeitos da fragmentação florestal em populações de Esenbeckia leiocarpa Engl. Scientia Forestalis, v. 57, p. 123-139, 2000.

SEOANE, C. E. S.; KAGEYAMA, P. Y.; RIBEIRO, A.; MATIAS, R.; REIS, M. S.; BAWA, K.; SEBBENN, A. M. Efeitos da fragmentação florestal sobre a imigração de sementes e a estrutura genética temporal de populações de Euterpe edulis M. Revista do Instituto Florestal, v. 17, n. 1, p. 23-43, 2005.

SIMBERLOFF, D.; COX, J. Consequences and costs of conservation corridors. Conservation Biology, v. 1, p. 63-71, 1987.

SIMBERLOFF, D. The contribution of population and community biology to conservation science. Annual Review of Ecology and Systematics .v. 19, p. 473-511, 1988. 
SJOGREN, R. D. Mosquito control: What does the future hold? Pest Control, v. 1, p. 10. 1991.

SLATKIN, M. An exact test for neutrality based on the ewens sampling distribution. Genetical Research .v. 64, n. 1, p. 71-74, 1994.

SOULÉ, M. E. Theory and strategy. Landscape Linkages and Biodiversity, 1991.

STEWART, F. M.; GORDON, D.M.; LEVIN, B.R. Fluctuation analysis: the probability-distribution of the number of mutants under different conditions. Genetics, v. 124, n. 1, p. 175-185, 1990.

TABARELLI, M.; GASCON, C. Lessons from fragmentation research: Improving management and policy guidelines for biodiversity conservation. Conservation Biology, v. 19, n. 3, p. 734-739, 2005.

TEMPLE, S. A.; CARY, J. R. Modeling dynamics of as an edge effect in habitat islands: experimental evidence habitat-interior bird populations in fragmented landscapes. Ecology, v. 69, p. 544 547,1988
VIEIRA, M. W.; CARAUTA, J. P. P.; DELGADO, W. A. Restauração de Áreas Semidegradadas Através da Implantação de Corredores Ecológicos. In: SIMPÓSIO NACIONAL SOBRE RECUPERAÇÃO DE ÁREAS DEGRADADAS, 5., 2002, Lavras. Anais. Lavras: UFLA, 2002. p.160-164.

WELDON, A. J. How corridors reduce Indigo Bunting nest success. Conservation Biology, v. 20, n. 4, p. 1300-1305, 2006.

WILCOVE, D. S.; MCLENNA, C. H.; DOBSON, A. P. Habitat fragmentation in the temperate zone. In: SOULÉ, M. E. (ed.):

Conservation Biology: the science of scarcity and diversity. Sinauer Inc. MA, USA, p. 237-56, 1986.

WILSON, E. O.; WILLIS, E. O. Applied biogeography. In: Ecological structure of species in communities. CODY, M. L.; DIAMOND, J. M. (eds.) Cambridge, Mass., Harvard University Press, p. 522-534, 1975.

YOUNG, A. G.; BOSHIER, D.; BOYLE, T. J. Forest

Conservation Genetics: principles and practice. CSIRO Publishing, 2000. p. 352

Recebido em 06 de novembro de 2010 e aprovado em 16 de agosto de 2010 